\title{
Enhancement of Automaticity by Mechanical Stretch of the Isolated Guinea Pig Pulmonary Vein Myocardium
}

\author{
Shogo Hamaguchi, ${ }^{* a}$ Koh Hikita ${ }^{a}$ Yusuke Tanaka, ${ }^{a}$ Yayoi Tsuneoka ${ }^{a, b}$ Iyuki Namekata, ${ }^{a}$ and \\ Hikaru Tanaka ${ }^{a}$ \\ ${ }^{a}$ Department of Pharmacology, Faculty of Pharmaceutical Sciences, Toho University; Funabashi, Chiba 274-8510, \\ Japan: and ${ }^{b}$ Laboratory of Pharmacology, Faculty of Pharmaceutical Sciences, Tokyo University of Science; Noda, \\ Chiba 278-8510, Japan.
}

Received December 14, 2015; accepted April 4, 2016

\begin{abstract}
The effects of mechanical stretch on the automaticity of the guinea pig isolated pulmonary vein preparations were studied in microelectrode experiments. The application of cumulative mechanical stretch to the preparations resulted in increases in the firing rate of spontaneous electrical activity in the myocardial layer. This effect was significantly inhibited by stretch-activated channel blockers such as gadolinium or streptomycin. These results suggest that acute mechanical stretch enhances the automaticity of the guinea pig pulmonary vein myocardium through the opening of the stretch-activated channels.
\end{abstract}

Key words pulmonary vein; spontaneous activity; mechanical stretch

The pulmonary vein contains a myocardial layer which is connected to the left atrial myocardium and is capable of generating spontaneous or triggered action potentials. ${ }^{1)}$ The finding that paroxysmal atrial fibrillation is initiated by trains of rapid discharges from the pulmonary veins, ${ }^{2)}$ has attracted great attention to the electrical activity of the pulmonary vein myocardium as a key player in the generation and maintenance of atrial fibrillation. ${ }^{3,4)}$ Microelectrode recordings from the myocardial layer have been performed in isolated pulmonary vein preparations from several experimental species, and difference in the firing pattern and frequency was observed. ${ }^{5}$ Studies including those from our laboratory have clarified that factors such as reduced repolarizing power and elevated intracellular calcium ion are involved in the manifestation of automaticity in the pulmonary vein myocardium. ${ }^{6}$ The electrical activity of the pulmonary vein myocardium was also shown to be affected by various neuronal and hormonal factors. However, less information is available concerning the relation between pathological condition and pulmonary vein automaticity.

The major risk factors of atrial fibrillation include highblood pressure, heart failure and valvular dysfunction; conditions which are accompanied by mechanical stretch of the heart. Chronic mechanical stretch of the heart induces histological remodeling of the heart such as eccentric hypertrophy and atrial enlargement. ${ }^{7)}$ Based on the understanding that the pulmonary vein automaticity serves as a trigger for the initiation of atrial fibrillation, it is probable that chronic mechanical stretch of the atrial region including the pulmonary vein increases the tendency to generate atrial fibrillation through long-term enhancement of the automaticity of the pulmonary vein myocardium. On the other hand, mechanical stretch may acutely affect cardiomyocytes through the opening of stretch-activated channels (SACs) and lead to the generation the trigger for atrial fibrillation. It was indeed reported in the isolated sheep heart that acute application of intra-atrial pressure increases the rate and organization of excitation waves emanating from the pulmonary veins. ${ }^{8)}$ It was also reported that the stretch-induced atrial fibrillation in perfused rabbit hearts was successfully inhibited by venom of the tarantula Grammostola spatulata which is a blocker of SACs. ${ }^{9)}$ These results led us to the notion that acute mechanical stretch might enhance the automaticity of pulmonary vein myocardium. In the present study, we found that acute mechanical stretch indeed increases the spontaneous firing rate of the isolated guinea pig pulmonary vein myocardium, and examined the effects of SAC blockers.

\section{MATERIALS AND METHODS}

All experiments were performed in accordance with the Guiding Principles for the Care and Use of Laboratory Animals approved by The Japanese Pharmacological Society and the Guide for the Care and Use of Laboratory Animals at Faculty of Pharmaceutical Sciences, Toho University.

The hearts with lungs were quickly removed from guinea pigs and preparations were made from the four major pulmonary vein trunks. The experimental procedures for microelectrode experiments were basically the same as those in our previous studies. ${ }^{6,10)}$ The region of the pulmonary vein close to the orifice was cut open and suspended in a $20 \mathrm{~mL}$ organ bath to record the tension in the circumferential direction. The physiological salt solution was of the following composition (mM): $\mathrm{NaCl} 137, \mathrm{KCl} 4.0, \mathrm{CaCl}_{2} 2.7, \mathrm{MgCl}_{2} 0.5, \mathrm{NaH}_{2} \mathrm{PO}_{4}$ 15.0 and glucose $11.0\left(\mathrm{pH} 7.4\right.$ ), aerated with $97 \% \mathrm{O}_{2}-3 \% \mathrm{CO}_{2}$ and maintained at $36 \pm 0.5^{\circ} \mathrm{C}$. One end of the preparation was pinned down on a silicon block at the bottom of the organ bath and the other end was attached to a needle connected to a force displacement transducer (TB-611T; Nihon Kohden, Tokyo, Japan).

Gadolinium chloride (Sigma-Aldrich, St. Louis, MO, U.S.A.) and streptomycin sulfate (Wako Pure Chemical Industries, Ltd., Osaka, Japan) were dissolved in distilled water. Small aliquots were added to the organ bath to obtain the desired final concentration. All other chemicals were commercial products of the highest available quality. All data are expressed as the mean \pm standard error of the mean (S.E.M.). Statistical significance between means was analyzed by two- 
A

a control

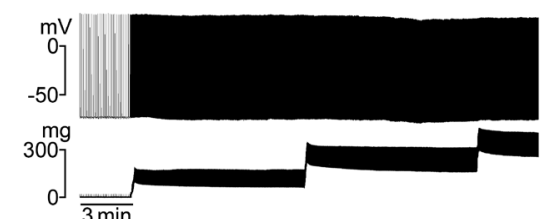

b gadolinium

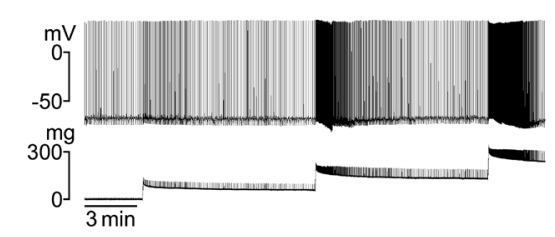

C streptomycin

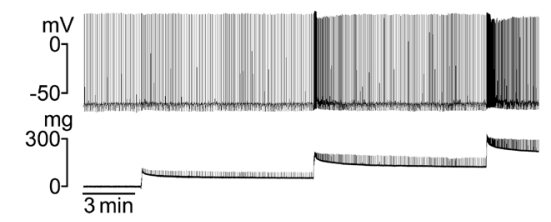

B
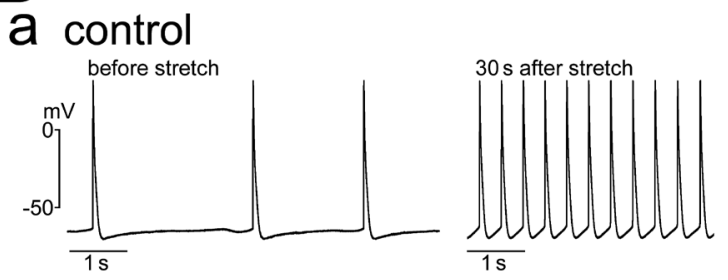

b gadolinium

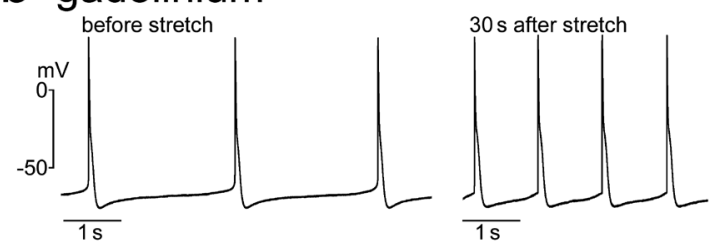

C streptomycin

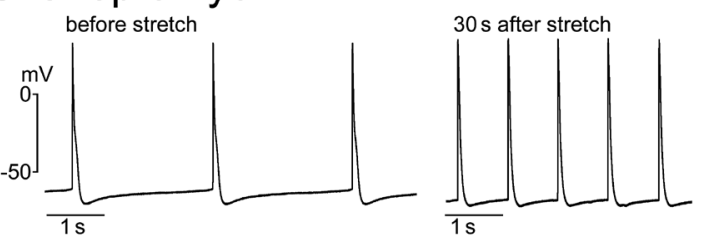

C

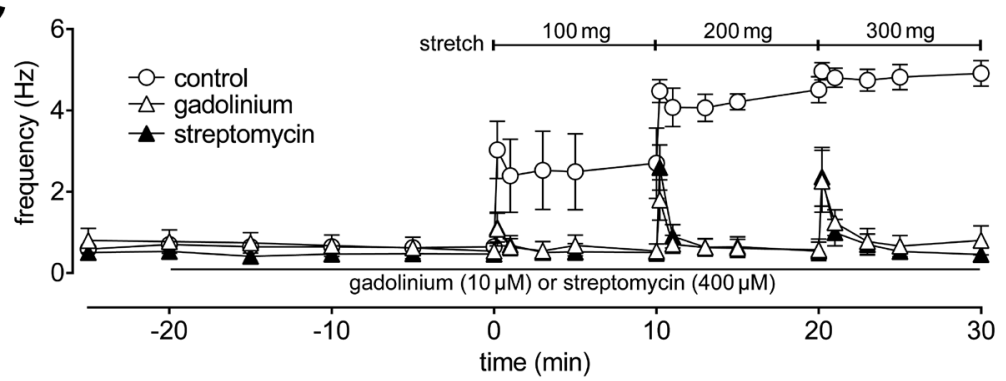

Fig. 1. Effects of Mechanical Stretch on the Spontaneous Electrical Activity of the Pulmonary Vein Myocardium

A: Typical traces of action potential (upper) and contraction (lower) showing the effect of incremental application of mechanical stretch in the absence (a) and presence of $10 \mu \mathrm{M}$ gadolinium (b) or $400 \mu \mathrm{m}$ streptomycin (c). B: Expanded traces obtained before (left) and $30 \mathrm{~s}$ after (right) the application of mechanical stretch (100 mg) in the absence (a) and presence of gadolinium (b) or streptomycin (c). C: Summarized results for the effects of mechanical stretch in the absence (open circles) and presence of $10 \mu \mathrm{M}$ gadolinium (open triangles) or $400 \mu \mathrm{M}$ streptomycin (closed triangles). Symbols are the mean \pm S.E.M. from 4 preparations. Concerning all data points after the application of mechanical stretch, the values obtained in the presence of SAC blockers were significantly different from corresponding values obtained in the absence.

way ANOVA with repeated measures followed by Bonferroni's test for multiple comparisons, and a $p$ value less than 0.05 was considered significant.

\section{RESULTS}

The incidence of spontaneous electrical activity in the guinea pig isolated pulmonary vein preparation was $67 \%$ $(46 / 68)$. In the absence of mechanical stretch, the frequency of spontaneous firing was $0.55 \pm 0.09 \mathrm{~Hz}(n=12)$. Application of mechanical stretch in incremental steps induced an incremental increase in the firing rate (Figs. 1Aa, Ba). The firing rate after application of stretch showed an initial peak phase with a duration of about $30 \mathrm{~s}$, which was followed by a plateau phase (Fig. 1C). The increase in firing rate was accompanied by an increase in the slope of the phase 4 depolarization (Fig. 1Ba, Table 1). Other parameters of the action potential were not affected by mechanical stretch (Table 1).

The stretch-activated channel blockers gadolinium $(10 \mu \mathrm{M})$ or streptomycin $(400 \mu \mathrm{M})$ had no effect on the spontaneous firing rate in the absence of mechanical stretch (Fig. 1C). The mechanical stretch-induced increase in firing rate was significantly inhibited by gadolinium (Fig. 1Ab) or streptomycin (Fig. 1Ac); the inhibition of the initial phase of increase was partial while the plateau phase was almost completely abolished by the blockers (Fig. 1C). The stretch-induced increase in firing rate at $30 \mathrm{~s}$ after application of $100 \mathrm{mg}$ tension was attenuated by gadolinium and streptomycin to $23.0 \pm 13.2 \%$ and $27.4 \pm 13.8 \%(n=4)$, respectively, of that in their absence, and that at $10 \mathrm{~min}$ to $0.5 \pm 1.3 \%$ and $2.1 \pm 2.5 \%(n=4)$, respectively. The inhibition of the increase in firing rate by SAC blockers was accompanied by an attenuation of the increase in the slope of phase 4 depolarization (Figs. 1Bb, c, Table 1).

\section{DISCUSSION}

In the present study, the incidence of spontaneous electrical activity in the myocardial layer of the isolated guinea pig 
Table 1. Effects of Mechanical Stretch on Myocardial Action Potential Parameters of the Guinea Pig Pulmonary Vein Preparations in the Absence and Presence of SAC Blockers

\begin{tabular}{|c|c|c|c|c|c|c|}
\hline & \multicolumn{2}{|c|}{ Control } & \multicolumn{2}{|c|}{ Gadolinium $(10 \mu \mathrm{M})$} & \multicolumn{2}{|c|}{ Streptomycin $(400 \mu \mathrm{M})$} \\
\hline & Before stretch & After $100 \mathrm{mg}$ stretch & Before stretch & After $100 \mathrm{mg}$ stretch & Before stretch & After $100 \mathrm{mg}$ stretch \\
\hline Take off potential $(\mathrm{mV})$ & $-70.7 \pm 6.1$ & $-70.9 \pm 6.1$ & $-65.4 \pm 5.0$ & $-62.9 \pm 4.7$ & $-60.8 \pm 3.6$ & $-57.3 \pm 2.0$ \\
\hline MDP $(\mathrm{mV})$ & $-79.3 \pm 3.2$ & $-76.6 \pm 3.5$ & $-71.8 \pm 4.3$ & $-67.8 \pm 3.9$ & $-69.9 \pm 2.0$ & $-66.1 \pm 0.7$ \\
\hline $\mathrm{AMP}(\mathrm{mV})$ & $97.4 \pm 7.1$ & $97.6 \pm 6.0$ & $97.1 \pm 3.7$ & $92.0 \pm 5.6$ & $89.2 \pm 2.1$ & $86.2 \pm 1.1$ \\
\hline $\mathrm{APD}_{20}(\mathrm{~ms})$ & $14.2 \pm 1.5$ & $14.3 \pm 0.8$ & $15.5 \pm 1.7$ & $16.4 \pm 1.5$ & $15.7 \pm 3.4$ & $15.7 \pm 4.5$ \\
\hline $\mathrm{APD}_{50}(\mathrm{~ms})$ & $34.8 \pm 2.0$ & $35.4 \pm 1.7$ & $31.1 \pm 3.0$ & $32.6 \pm 2.5$ & $30.7 \pm 4.5$ & $29.7 \pm 5.4$ \\
\hline $\mathrm{APD}_{90}(\mathrm{~ms})$ & $103.2 \pm 3.9$ & $94.1 \pm 4.8$ & $96.0 \pm 5.3$ & $92.3 \pm 6.5$ & $103.1 \pm 11.0$ & $88.3 \pm 3.4$ \\
\hline$\dot{V}_{\max }(\mathrm{V} / \mathrm{s})$ & $119.8 \pm 41.1$ & $112.2 \pm 36.3$ & $77.2 \pm 21.3$ & $66.0 \pm 17.7$ & $68.8 \pm 20.5$ & $61.8 \pm 16.6$ \\
\hline Slope $(\mathrm{mV} / \mathrm{s})$ & $4.7 \pm 1.0$ & $27.9 \pm 5.3^{*}$ & $3.3 \pm 1.2$ & $6.8 \pm 3.3^{\dagger}$ & $3.2 \pm 1.4$ & $9.6 \pm 4.3^{\dagger}$ \\
\hline
\end{tabular}

MDP, AMP, $\dot{V}_{\max }$ and slope indicate maximum diastolic potential, amplitude, maximum rate of phase 0 depolarization and maximum rate of phase 4 depolarization, respectively. $\mathrm{APD}_{20}, \mathrm{APD}_{50}$, and $\mathrm{APD}_{90}$ indicate action potential duration at 20,50 , and $90 \%$ repolarization, respectively. Values are the mean $\pm \mathrm{S} . \mathrm{E} . \mathrm{M}$. from 4 pulmonary vein preparations. Asterisks and daggers indicate a significant difference from corresponding values before stretch and in the control, respectively.

pulmonary vein preparations was higher than those reported in other small experimental animals such as the rabbit ${ }^{11)}$ and rat. $^{12)}$ Thus, the guinea pig appears to be an important animal for studies on the spontaneous electrical activity of the pulmonary vein myocardium. Concerning the high incidence of spontaneous electrical activity in the absence of mechanical stretch, mechanisms other than activation of SACs are certainly involved, because neither gadolinium nor streptomycin, blockers of the SACs, affected the spontaneous firing. This also suggests that these blockers did not affect the $\mathrm{Na}^{+}, \mathrm{Ca}^{2+}$ and $\mathrm{K}^{+}$channels involved in the basic electrical activity of the myocardium under the present experimental condition.

The main finding of the present study is that mechanical stretch on the guinea pig pulmonary vein results in enhancement of spontaneous automaticity of the pulmonary vein myocardium through activation of SACs which contributes to the phase 4 depolarization. Application of mechanical stretch had no significant effect on other parameters of the action potential waveform which suggests that the $\mathrm{Na}^{+}, \mathrm{Ca}^{2+}$ and $\mathrm{K}^{+}$channels responsible for the rapid depolarization and repolarization of the myocardium were not affected. Gadolinium and streptomycin, blockers of the SACs which attenuated the stretchinduced increase in firing rate, did not affect the basal firing rate in the absence of stretch. This suggests that the SACs provide an enhancing mechanism which works in addition to the basal firing mechanism. Stretch-induced enhancement of automaticity and its inhibition by SAC blockers was also reported in the isolated rabbit pulmonary vein. ${ }^{11)}$ These results suggest that SACs are involved in the automaticity of the pulmonary vein myocardium in various animal species and possibly in humans. Studies on the effects of various antiarrhythmic agents on the guinea pig pulmonary vein myocardium is now in progress. ${ }^{13)}$ The present study raises the possibility that the action of pharmacological agents are partially mediated by their effects on SACs.

The SACs include a variety of channels which belong to several different channel families. ${ }^{14)}$ Expression of SAC types such as the non-selective cation channel and the 4,4'-diisothiocyano-2,2'-stilbenedisulfonic acid (DIDS)-sensitive chloride channel has been reported in rabbit pulmonary vein cardiomyocytes. ${ }^{15)}$ As the blocking effect of gadolinium and streptomycin are not selective, the molecular identity of the channel involved in the automaticity of the guinea pig pulmonary vein myocardium remains to be investigated. It is also possible that cells such as the smooth muscle cell and endothelial cell are involved in the response to mechanical stretch. The observation that the inhibitory potency of both gadolinium and streptomycin was different between the early and late phases after application of mechanical stretch may reflect the presence of multiple mechanisms.

A common feature of SACs is that their openings cause depolarization of the cell membrane. ${ }^{14)}$ This provides a probable mechanism for stretch-induced enhancement of automaticity. The depolarizing power can enhance the phase 4 depolarization of the pulmonary vein myocardium and lead to an increase in firing rate. The present results that the slope of the phase 4 depolarization was increased after application of mechanical stretch, and that it was attenuated by gadolinium and streptomycin provide evidence for such view. Earlier reports showed that the repolarizing power of the pulmonary vein myocardium is less than that of the working myocardium, ${ }^{6}$ ) which would play a permissive role in the manifestation of such mechanism. On the other hand, several reports, including those from our laboratory, showed that the automaticity of the pulmonary vein myocardium is dependent on intracellular calcium ion. ${ }^{45)}$ In the guinea pig pulmonary vein myocardium, constant electrical firing was enhanced by interventions which increase intracellular calcium ion such as repetitive electrical stimulation ${ }^{16)}$ and ouabain treatment. ${ }^{6)}$ Thus, another probable mechanism for the enhancement of automaticity by mechanical stretch is the increase in supply of calcium ion to the cytoplasm. Thus, whether SACs contribute to the diastolic depolarization as a carrier of depolarizing current or rather as a supply route to increase intracellular $\mathrm{Ca}^{2+}$ remains to be investigated.

Conditions accompanied with mechanical stretch of the heart such as high-blood pressure, heart failure and valvular dysfunction, are known to be risk factors for atrial fibrillation. ${ }^{8)}$ Tendency towards atrial fibrillation has been reported in several animal models accompanied by mechanical stretch of the atria. ${ }^{17)}$ Concerning the involvement of the pulmonary vein, we have reported in the atrio-venocaval shunt rat, which is characterized by mechanical stretch of the atrial region, that the pulmonary vein myocardium acquires an enhanced automaticity and an increased tendency towards atrial fibrillation after chronic mechanical stretch. ${ }^{10)}$ The results of the present study suggested that acute mechanical stretch to the pulmonary vein enhances the automaticity of pulmonary vein 
myocardium through opening of SACs, which can probably serve as a trigger for atrial fibrillation. To clarify whether this mechanism indeed operates in the pulmonary vein in vivo, further experiments including measurement of blood pressure of the pulmonary circulation and in vivo recording of electrical activity from the pulmonary vein are needed. The blood pressure of the pulmonary circulation as well as the level of autonomic and hormonal influence are known to be altered under various pathological conditions. ${ }^{4,5,13)}$ Whether blockers of SACs are effective against various models of atrial fibrillation is a matter of great interest.

Acknowledgments This study was supported in part by Grants-in-Aid from the Ministry of Education, Culture, Sports, Science and Technology of Japan to I.N. (\#25860194), and H.T. (\#15K08247).

Conflict of Interest The authors declare no conflict of interest.

\section{REFERENCES}

1) Tasaki H. Electrophysiological study of the striated muscle cells of extrapulmonary vein of guinea-pig. Jpn. Circ. J., 33, 1087-1098 (1969).

2) Haïssaguerre M, Jaïs P, Shah DC, Takahashi A, Hocini M, Quiniou G, Garrigue S, Le Mouroux A, Le Métayer P, Clémenty J. Spontaneous initiation of atrial fibrillation by ectopic beats originating in the pulmonary veins. N. Engl. J. Med., 339, 659-666 (1998).

3) Chen YJ, Chen SA, Chang MS, Lin CI. Arrhythmogenic activity of cardiac muscle in pulmonary veins of the dog: implication for the genesis of atrial fibrillation. Cardiovasc. Res., 48, 265-273 (2000).

4) Nattel S. Basic electrophysiology of the pulmonary veins and their role in atrial fibrillation. $J$. Cardiovasc. Electrophysiol., 14, 13721375 (2003).

5) Namekata I, Tsuneoka Y, Tanaka H. Electrophysiological and pharmacological properties of the pulmonary vein myocardium. Biol. Pharm. Bull., 36, 2-7 (2013).

6) Namekata I, Tsuneoka Y, Takahara A, Shimada H, Sugimoto T, Takeda K, Nagaharu M, Shigenobu K, Kawanishi T, Tanaka H.
Involvement of the $\mathrm{Na}^{+} / \mathrm{Ca}^{2+}$ exchanger in the automaticity of guinea-pig pulmonary vein myocardium as revealed by SEA0400. J. Pharmacol. Sci., 110, 111-116 (2009).

7) De Jong AM, Maass AH, Oberdorf-Maass SU, Van Veldhuisen DJ, Van Gilst WH, Van Gelder IC. Mechanisms of atrial structural changes caused by stretch occurring before and during early atrial fibrillation. Cardiovasc. Res., 89, 754-765 (2011).

8) Kalifa J, Jalife J, Zaitsev AV, Bagwe S, Warren M, Moreno J, Berenfeld $\mathrm{O}$, Nattel S. Intra-atrial pressure increases rate an organization of waves emanating from the superior pulmonary veins during atrial fibrillation. Circulation, 108, 668-671 (2003).

9) Bode F, Sachs F, Franz MR. Tarantula peptide inhibits atrial fibrillation. Nature, 409, 35-36 (2001).

10) Hamaguchi $S$, Tsuneoka $Y$, Tanaka A, Irie $M$, Tsuruta $M$, Nakayama T, Namekata I, Nada M, Aimoto M, Takahara A, Tanaka H. Manifestation of automaticity in the pulmonary-vein myocardium of rats with abdominal aorto-venocaval shunt. J. Pharmacol. Sci., 128, 212-215 (2015).

11) Chang SL, Chen YC, Chen YJ, Wangcharoen W, Lee SH, Lin CI, Chen SA. Mechanoelectrical feedback regulates the arrhythmogenic activity of pulmonary veins. Heart, 93, 82-88 (2007).

12) Maupoil V, Bronquard C, Freslon JL, Cosnay P, Findlay I. Ectopic activity in the rat pulmonary vein car arise from simultaneous activation of $\alpha 1$ - and $\beta 1$-adrenoceptors. Br. J. Pharmacol., 150, 899-905 (2007).

13) Takahara A, Hagiwara M, Namekata I, Tanaka H. Pulmonary vein myocardium as a possible pharmacological target for the treatment of atrial fibrillation. J. Pharmacol. Sci., 126, 1-7 (2014).

14) Ninio DM, Saint DA. The role of stretch-activated channels in atral fibrillation and the impact of intracellular acidocis. Prog. Biophys. Mol. Biol., 97, 401-416 (2008).

15) Seol CA, Kim WT, Ha JM, Choe H, Jang YJ, Youm JB, Earm YE, Leem CH. Stretch-activated currents in cardiomyocytes isolated from rabbit pulmonary veins. Prog. Biophys. Mol. Biol., 97, 217-231 (2008).

16) Takahara A, Sugimoto $T$, Kitamura $T$, Takeda K, Tsuneoka $Y$, Namekata I, Tanaka H. Electrophysiological and pharmacological characteristics of triggered activity elicited in guinea-pig pulmonary-vein myocardium. J. Pharmacol. Sci., 115, 176-181 (2011).

17) Nishida K, Michael G, Dobrev D, Nattel S. Animal models for atrial fibrillation: clinical insights and scientific opportunities. Europace, 12, 160-172 (2010). 